\title{
Deliberate employment of postoperative hypotension for brain arteriovenous malformation surgery and the incidence of delayed postoperative hemorrhage: a prospective cohort study
}

\author{
Michael Kerin Morgan, MD, ${ }^{1}$ Markus Karl Hermann Wiedmann, MD, ${ }^{1}$ \\ Nazih N. A. Assaad, MB, BS, FRACS, ${ }^{1}$ Michael J. A. Parr, MBBS, ${ }^{1,3}$ and Gillian Z. Heller, PhD² \\ Departments of ${ }^{1} \mathrm{Clinical}$ Medicine and ${ }^{2}$ Statistics, Macquarie University, Sydney; and ${ }^{3}$ Department of Intensive Care, Macquarie \\ University Hospital, Ryde, New South Wales, Australia
}

\begin{abstract}
OBJECTIVE The aim of this study was to examine the impact of deliberate employment of postoperative hypotension on delayed postoperative hemorrhage (DPH) for all Spetzler-Ponce Class (SPC) $\mathrm{C}$ brain arteriovenous malformations (bAVMs) and SPC B bAVMs $\geq 3.5 \mathrm{~cm}$ in diameter (SPC B 3.5+).
\end{abstract}

METHODS A protocol of deliberate employment of postoperative hypotension was introduced in June 1997 for all SPC $\mathrm{C}$ and SPC B 3.5+ bAVMs. The aim was to achieve a maximum mean arterial blood pressure (BP) $\leq 70 \mathrm{~mm} \mathrm{Hg}$ (with cerebral perfusion pressure $>50 \mathrm{~mm} \mathrm{Hg}$ ) for a minimum of 7 days after resection of bAVMs (BP protocol). The authors compared patients who experienced DPH (defined as brain hemorrhage into the resection bed that resulted in a new neurological deficit or that resulted in reoperation during the hospitalization for microsurgical bAVM resection) between 2 periods (prior to adopting the BP protocol and after introduction of the BP protocol) and 4 bAVM categories (SPC A, SPC B 3.5- [that is, SPC B $<3.5 \mathrm{~cm}$ maximum diameter], SPC B 3.5+, and SPC C). Patients excluded from treatment by the $\mathrm{BP}$ protocol were managed in the intensive care unit to avoid moderate hypertensive episodes. The pooled cases of all bAVM treated by surgery were analyzed to identify characteristics associated with the risk of DPH. These identified characteristics were then examined by multiple logistic regression analysis in both SPC B 3.5+ and SPC C cases.

RESULTS From a cohort of 641 bAVMs treated by microsurgery, 32 patients with DPH were identified. Of those, $66 \%$ $(95 \% \mathrm{Cl} 48-80)$ had a permanent new neurological deficit with a modified Rankin Scale score of $2-6$. This included a mortality rate of $13 \%(95 \% \mathrm{Cl} 4.4-29)$. The BP protocol was used to treat 162 patients with either SPC B 3.5+ or SPC C. For SPC B 3.5+, there was no significant reduction in DPH with the introduction of the BP protocol $(p=0.77)$. For SPC $\mathrm{C}$, there was a significant $(\mathrm{p}=0.035)$ reduction of $\mathrm{DPH}$ from $29 \%(95 \% \mathrm{Cl} 13 \%-53 \%)$ to $8.2 \%(95 \% \mathrm{Cl} 3.2 \%-18 \%)$ associated with the introduction of the BP protocol. Multiple logistic regression analysis found that the absence of the BP protocol ( $p=0.011$, odds ratio $7.5,95 \% \mathrm{Cl} 1.6-36$ ) remained significant for the development of DPH in patients with SPC C bAVMs.

CONCLUSIONS Treating patients with SPC C bAVMs with a protocol that lowers BP immediately after resection seems to reduce the risk of DPH. For SPC A and SPC B 3.5- bAVMs, there is unlikely to be a need to do more than avoid postoperative hypertension. For SPC B 3.5+ bAVMs, a larger number of patients would be required to test the absence of benefit of the BP protocol.

https://thejns.org/doi/abs/10.3171/2016.9.JNS161333

KEY WORDS brain; arteriovenous malformation; AVM; surgery; intensive care; blood pressure; hemorrhage; cohort study; vascular disorders 
$\mathrm{O}$ $\mathrm{NE}$ of the feared consequences of brain arteriovenous malformation (bAVM) surgery is delayed postoperative hemorrhage (DPH). There are many causes for DPH, including both those associated with general intradural brain surgery as well as those unique to bAVM surgery. The latter are related to the altered hemodynamics resulting from the rapid abolition of an arteriovenous fistula. This group of hemodynamically induced hemorrhage, first reported in 1977 by Spetzler and colleagues, ${ }^{33}$ may be caused by normal perfusion pressure breakthrough, venous occlusive hyperemia, a retained nidus, or rupture

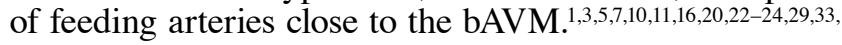
34,37

A unifying mechanism for these various points of vascular vulnerability responsible for DPH is arteriocapillaryvenous hypertension syndrome due to the rise in pressures proximal to the point of arteriovenous fistula ablation in a dilated proximal vascular bed, irrespective of the site of this occlusion. ${ }^{19}$ From series in which many surgical cases were performed in a period predating the wide adoption of aggressive and systematic blood pressure (BP) control, the incidence of DPH (or complications that are attributed to hemodynamic consequences of removing the arteriovenous fistula) has ranged from $2 \%$ to $21 \%$., $4,11,16,18,19,24,36$ However, estimating the incidence is inexact due to case selection and the adoption of strategies that were deemed likely to reduce the risk of DPH. ${ }^{2,10,31}$

Because a common physiological mechanism includes a focal elevation of pressures within and around the bAVM resection bed, an approach to DPH avoidance includes BP control..$^{10,32}$ Furthermore, there is evidence suggesting a benefit for lowering the BP below normal for selected patients after bAVM resection. ${ }^{21}$ This was first reported to be successful in clinical practice to reverse normal perfusion pressure breakthrough by Day and colleagues in 1982. ${ }^{10}$ However, controversy exists as to whether such strategies are necessary in view of the inherent vigilance required to avoid complications and the resources necessary to implement this management strategy. 25

A simplification of the Spetzler-Martin grading system, which has been validated to predict the risk of surgery, combines Spetzler-Martin Grades (SMGs) I and II as Spetzler-Ponce Class (SPC) A bAVMs (SPC A), SMG III as SPC B bAVMs (SPC B), and SMGs IV and V as SPC C bAVMs (SPC C). ${ }^{32}$ Following bAVM surgery, all patients were managed by the senior author (M.K.M.) in intensive care units (ICUs) for the purpose of preventing hypertension. However, in June 1997, a more aggressive approach was adopted with the introduction of a strict ICU protocol for the first 7 days after bAVM resection for SPC C as well as for SPC B with maximum diameter $\geq 3.5 \mathrm{~cm}$ (SPC $\mathrm{B} 3.5+$ ) for the purpose of lowering the mean arterial $\mathrm{BP}$ (MABP) to not exceed $70 \mathrm{~mm} \mathrm{Hg.}{ }^{17,21}$

From our initial reports, patients with SPC A and patients with SPC B 3.5- bAVM did not develop DPH. The aim of this study was to examine the benefits with regard to reducing the incidence of DPH and determining for whom this protocol is best suited. In addition, we sought to examine whether patients excluded from the BP protocol (those with SPC A and SPC B 3.5-) developed DPH and were appropriately excluded from the BP protocol.

\section{Methods \\ Ethical Statement}

This study was approved by the institutional human ethics committee and was performed in accordance with institutional ethics committee guidelines.

\section{Data Collection and Study Cohort}

The senior author prospectively collected a bAVM database. This database has been previously described. ${ }^{9,14}$ The senior author was responsible for clinical and imaging assessment as well as entry into the database. However, the database was accessible to residents, fellows, and occupational therapists at the time of assessment and follow-up, and details of the database entries could be examined. The database contained demographic, clinical, imaging, and treatment-related information.

Patients were included in the database if they were confirmed to have a bAVM on MRI, CT angiography (CTA), or digital subtraction angiography (DSA). The SMG was allocated preoperatively when sufficient imaging details had been obtained to grade the bAVM. An adverse outcome from surgery was defined as a new permanent neurological deficit (assigned within the first 6 weeks of surgery) with a modified Rankin Scale (mRS) score $>1,{ }^{26,35}$ assigned at 12 months after surgery due to preoperative embolization or surgery. This database captured patient data from 1989 until May 2014 (the last month that surgery was conducted by the senior author).

Characteristics examined included patient demographic data (sex and age), bAVM characteristics (infratentorial location, maximum diameter, eloquent location, deep venous drainage, SMG, and anterior or posterior circulation supply), clinical data (preoperative mRS score and mode of presentation), treatment-related data (preoperative embolization and BP protocol period), and outcome-related data (mRS score, complications of surgery, presentation of $\mathrm{DPH}$, and length of follow-up).

\section{Outcome Characteristics Examined}

DPH was defined as a postoperative hemorrhage into the bAVM bed following bAVM resection, occurring prior to discharge from the hospital and resulting in a neurological decline or reoperation for evacuation.

\section{BP Protocol and Perioperative Management}

The BP protocol, which was previously published, was maintained from June 1997 until 2014. ${ }^{21}$ Because of the highly context-specific nature of ICU protocols, we have included the protocol in detail in the following paragraphs. For patients who were not managed specifically by the BP protocol, postoperative management took place in an ICU with emphasis on prevention of hypertension (defined as a systolic BP > $140 \mathrm{~mm} \mathrm{Hg}$ ). All patients were treated with preoperative and postoperative dexamethasone and anticonvulsant medications. Occasionally, large bAVMs were managed with aggressive antihypertensive therapy following surgery, prior to the implementation of the BP protocol, but this was not done with the same rigor or consistency as the BP protocol. 
The BP protocol ensured that all patients with SPC B 3.5+ bAVMs and all patients with SPC C bAVMs were treated in the ICU for a minimum of 7 days, during which arterial BP was strictly controlled. Intracranial pressure (ICP) measurements were obtained using either a subdural or ventricular catheter following surgery; this was only discontinued when the ICP was normal in the presence of Glasgow Coma Scale Score 15. BP control was initiated by preoperative loading with oral beta-blockers, the administration of which was continued in the postoperative period. Commencing with surgery, each patient's MABP was limited to a maximum of $70 \mathrm{~mm} \mathrm{Hg}$.

To achieve these BP protocol goals, the patient's management is more complex than it is for patients with SPC $A$ and SPC B 3.5- (in whom the aim is to avoid a systolic BP > $140 \mathrm{~mm} \mathrm{Hg}$ ). First, the patient managed with the BP protocol is usually intubated and ventilated for a greater length of time than is required solely for surgical anesthesia. This is to ensure that on emergence, BP can be restricted to an MABP $<70 \mathrm{~mm} \mathrm{Hg}$.

Second, the continuous management of BP necessitates multiple-lumen central venous access to ensure that titratable agents, such as sodium nitroprusside and alpha adrenergic agents (both are needed to precisely maintain $\mathrm{BP}$ within specified boundaries), are given in separate lines to optimize control and to prevent an inadvertent bolus of agents (passively occupying the line from previous administrations) that may produce the opposite effect on $\mathrm{BP}$ than intended.

In our experience, it can be extremely difficult to produce lower than normal BP in young patients, even though they normally have lower BP than older patients. Prolonged anesthesia may assist with achieving this goal in young patients if lowering their BP proves to be refractory with other measures. This degree of invasive management is usually not necessary for patients who are not managed with the BP protocol.

Following surgery, if the patient's cerebral perfusion pressure (CPP; defined, for the purpose of the protocol, as MABP-ICP) decreased to $<60 \mathrm{~mm} \mathrm{Hg}$, CT or CTA was performed at the MABP maximum limit of $70 \mathrm{~mm} \mathrm{Hg}$ to exclude the presence of a DPH. If DPH with mass effect was identified, surgery was performed. In the event that a minimum CPP of $60 \mathrm{~mm} \mathrm{Hg}$ could not be obtained and there was no surgically treatable space-occupying lesion, the patient was treated by induction of a therapeutic coma with sodium thiopental to a burst suppression pattern on an electroencephalogram. Once burst suppression was achieved, a CPP of $50 \mathrm{~mm} \mathrm{Hg}$ was accepted. Our method of barbiturate-induced coma has been previously described ${ }^{8}$

If $>1 \mathrm{~g}$ of sodium thiopental was used intraoperatively, or if the anesthesiologist believed that BP stability could not be achieved with extubation, the patient was transferred to the ICU and ventilated. The timing of extubation was determined to occur when BP and neurological stability were considered optimal. An escalating drug regimen was used to achieve the target BP. The regimen included the use of a beta-blocker, sodium nitroprusside, oral nimodipine, and an angiotensin-converting enzyme inhibitor. Invasive or potentially painful procedures (e.g., insertion of central lines) were permitted only with the supervision of experienced ICU staff members, and attention was given to maintaining BP restrictions. Fluid balance was carefully monitored and furosemide was used if a significant positive balance developed. Anticonvulsants were commenced prior to surgery to reduce the potential of seizures as well as seizure-related increases in BP.

Postoperative DSA was scheduled to be routinely performed on, or close to, Day 7 after surgery. This timing ensured that bAVMs that may have been only temporarily obliterated at the time of the resection had time to refill and that remodeling of the vasculature could be assessed. However, in patients with DPH who did not require urgent return to the operating room or in patients in whom postoperative Day 1 CTA suggested a residual bAVM, early DSA was performed. In patients experiencing DPH or in patients who were operated on for a residual bAVM, DSA was performed within 24 hours of these events.

\section{Inclusion and Exclusion Criteria}

All patients undergoing resection of bAVM were examined. We have previously demonstrated the significant impact of SMG on outcome of surgery in our cohort and have applied the BP protocol to a specific group of patients (those with SPC B 3.5+ and SPC C bAVMs). The surgical bAVM cohort was therefore divided into 4 groups according to bAVM classification (SPC A, SPC B 3.5-, SPC B $3.5+$, and SPC C) and analyzed in 2 epochs, that is, before and after implementation of the BP protocol. ${ }^{12}$

The reason for this a priori stratification was the significant impact that SMG had on outcome of surgery in our cohort, ${ }^{14}$ and the exclusion for application of the BP protocol in specific groups of patients (SPC A and SPC B 3.5-). ${ }^{21}$

Although not managed by the BP protocol, patients with SPC A and SPC B 3.5- bAVMs were included in the analyses to examine the incidence of DPH in these groups. However, they were not included in the multiple logistic regression analyses, the purpose of which was to examine characteristics associated with the risk of DPH for patients with SPC B 3.5+ and SPC C bAVMs, each managed by the BP protocol. The stratification of lesions into SPC A, SPC B 3.5-, SPC B 3.5+, and SPC C was made a priori due to previous evidence that stratification by SPC is an important predictor of the risk of surgery and biases in selecting patients for surgery. In addition, patients with SPC B 3.5+ bAVMs were managed by the BP protocol, whereas those with SPC B 3.5- bAVMs were not.

\section{Analysis of Baseline Characteristics Between SPC Groups and Between Epochs}

Patient and bAVM characteristics were included and compared to identify unexpected differences or similarities between groups (SPC A, SPC B 3.5-, SPC B 3.5+, and SPC C) and epochs (prior to BP protocol and during the $\mathrm{BP}$ protocol period).

\section{Analysis of Variables Influencing DPH}

The baseline characteristics, stratified into the 4 groups (SPC A, SPC B 3.5-, SPC B 3.5+, and SPC C bAVMs) with the 2 epochs (prior to BP protocol and during the 
BP protocol period), were examined for differences in the incidence of DPH.

From differences between patients who did and did not experience a DPH, variables chosen for the regression analyses were characteristics that were identified preoperatively to be significantly associated with DPH, with a maximum significance of $1 \%$. These included maximum diameter of the bAVM (as a continuous variable), eloquent location (as defined by Spetzler and Martin to include locations in the primary sensory cortex, motor cortex, language cortex, internal capsule, diencephalon, brainstem, deep cerebellar nuclei, or cerebellar peduncle), ${ }^{30}$ and concurrent supply from the anterior and posterior circulations (vs supply from a single circulation). The period of BP control (before or after BP protocol) was a variable selected on an a priori assumption. Because the regression analysis was separated into SPC B 3.5+ and SPC C, SPC was not selected as a variable, although its constituent contributing variables were selected.

Although diffuse bAVM has also been identified as a risk factor for complications of surgery and may be very relevant to the risk of $\mathrm{DPH},{ }^{13,15}$ this variable had not been collected prospectively in the database and therefore was not included in the analysis.

\section{Examination of the Appropriateness of the A Priori Decision to Use a Cut-Point of $3.5 \mathrm{~cm}$ Maximum bAVM Diameter}

A receiver operating characteristic (ROC) curve examined size and the development of DPH for the entire surgical cohort. The size maximizing the sum of sensitivity and specificity was considered the optimal cut-point.

\section{Statistical Analysis}

Comparisons between groups were performed using a $\chi^{2}$ test, Fisher exact test, Student t-test, and Mann-Whitney U-test, as appropriate. From the variables selected for analysis, multiple logistic regression analyses (backward Wald) were performed. Because of the large number of characteristics examined, a statistical significance level of $1 \%$ was used to select variables for the multiple logistic regression. For the multiple logistic regression analysis, as well as the a priori-examined group comparisons (that is, SPC A, SPC B 3.5-, SPC B 3.5+, and SPC C compared before and during the period of the BP protocol), a statistical significance level of 5\% was used. The modified Wald method was used to calculate the $95 \%$ CI for a proportion of the outcome data (www.graphpad.com/quickcalcs/). Statistical analysis was performed using Prism (version 6, GraphPad Software, Inc.) and IBM SPSS Statistics (version 22, IBM Corporation) software.

\section{Results}

From a database of 779 patients with bAVM, 642 underwent microsurgery for bAVM resection. One of these patients was excluded from the analysis, bringing the population to 641 patients. Although the surgery for an SPC B 3.5+ bAVM was performed during the period of the $\mathrm{BP}$ protocol, this one patient was not managed using the $\mathrm{BP}$ protocol. This patient was treated in a foreign hospital without an infrastructure capable of implementing the BP protocol (Fig. 1).

\section{Differences and Similarities in Patients Before and After Introduction of the BP Protocol}

The baseline characteristics and comparisons between patients treated before and after introduction of the BP protocol for postoperative bAVM management are provided in Table 1. Of note, there was a difference in the proportion of cases with deep venous drainage and eloquent locations between these 2 epochs (for SPC A). There was also a difference in the distribution of preoperative $\mathrm{mRS}$ scores between the 2 epochs, consistent with the greater number of asymptomatic patients treated during the period of the BP protocol. However, there was no difference in maximum diameter nor in the distribution of cases by the 4 a priori-analyzed categories (SPC A, SPC B 3.5-, SPC B 3.5+, and SPC C).

Treatment with preoperative embolization differed between the 2 periods for SPC A and SPC B 3.5- bAVMs (although not between SPC B 3.5+ and SPC C bAVMs).

There was no difference in the overall incidence of complications leading to a neurological deficit $>1$ at 12 months, nor in the distribution of the mRS scores between the 2 epochs.

\section{Differences and Similarities in Patients Between SPC B $3.5+$ and SPC C}

Differences expected of the separation into SPC B 3.5+ and SPC C were evident with respect to maximum diameter, deep venous drainage, and eloquent location. As expected, there was a difference in the proportion of patients who underwent embolization, particularly during the second period, with more patients with SPC C bAVMs undergoing embolization than those with SPC B 3.5+ bAVMs.

\section{Incidence of DPH}

Prior to introduction of the BP protocol, the overall incidence of DPH was 6.6\% (95\% CI 3.6-11), and during the period of the BP protocol, the incidence of DPH was $4.4 \%$ (95\% CI 2.9-6.7). The difference was not statistically significant $(\mathrm{p}=0.30)$. The 4 groups analyzed on an a priori basis before and after introduction of the $\mathrm{BP}$ protocol were SPC A, SPC B 3.5-, SPC B 3.5+, and SPC C (Fig. 1). The 2 groups SPC A and SPC B 3.5- were analyzed before and after introduction of the BP protocol (these patients were not subject to the BP protocol). The 2 groups SPC B $3.5+$ and SPC C were analyzed before and after introduction of the BP protocol (these patients were managed by the $\mathrm{BP}$ protocol).

For the 166 patients who underwent microsurgical resection of bAVM prior to the BP protocol, the incidence of DPH for each group was as follows: SPC A, $1 \%(95 \%$ CI $<0.01 \%-6.1 \%$ ); SPC B 3.5-, $0 \%$ (95\% CI 0\%-28\%); SPC B $3.5+, 12 \%$ (95\% CI $5.1 \%-27 \%)$; and SPC C, $29 \%(95 \%$ CI 5\%-17\%) (Table 1). For the 162 patients who underwent microsurgical resection of a bAVM and were managed with the BP protocol, the incidence of DPH was as follows: SPC B 3.5+, 11\% (95\% CI 6\%-19\%); and SPC C, $8.2 \%$ (95\% CI $3.2 \%-18 \%$ ) (Table 1). 


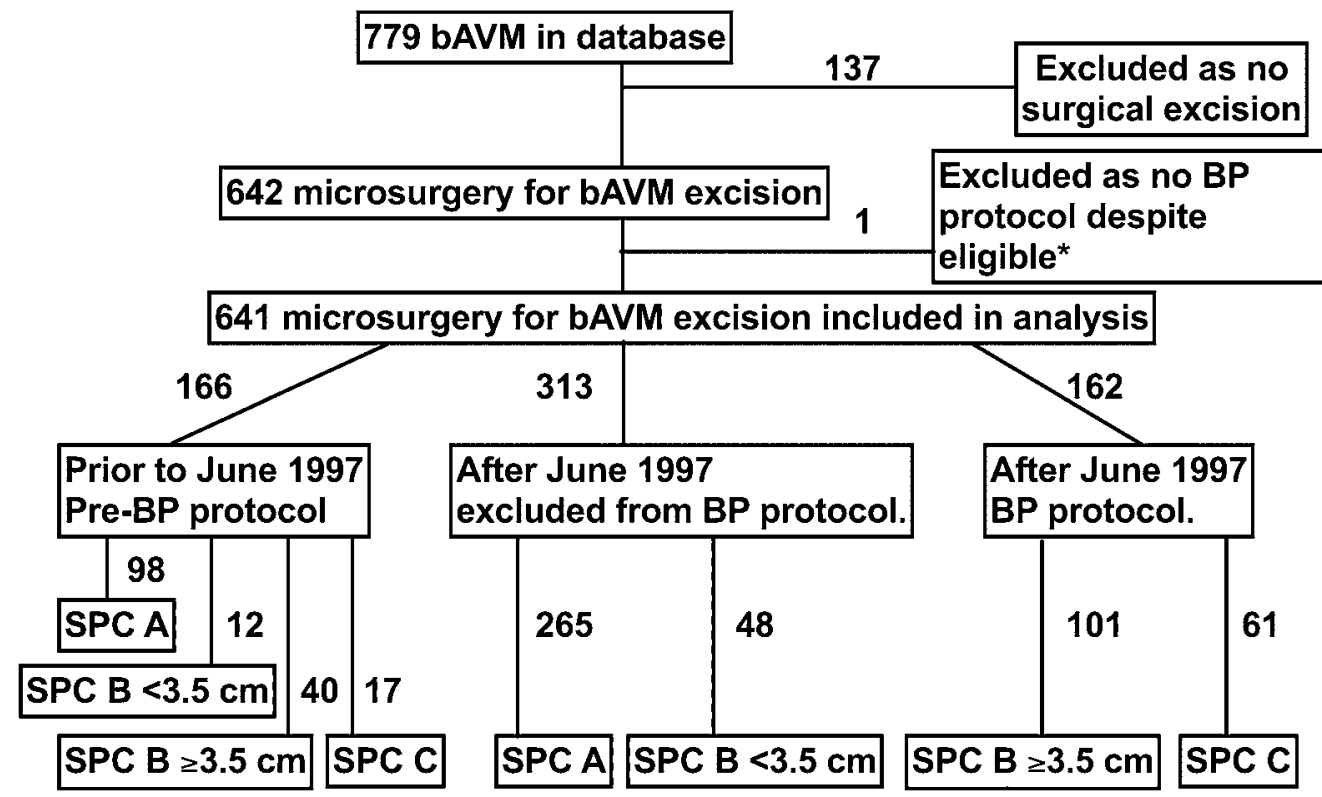

FIG. 1. Distribution of patients by the class of their bAVM and management by the BP protocol. The numbers indicate the number of patients in each group. *This excluded patient, who had an SPC B 3.5+ AVM, underwent surgery during the BP protocol period. However, this patient was not managed using the BP protocol because it was not possible in the ICU at the overseas hospital where treatment was received.

During this same period, during which the BP protocol was used, 313 patients were not managed by the BP protocol. For these patients, the incidence of DPH was as follows: SPC A, $1.5 \%$ (95\% CI 0.5\%-4.0\%); and SPC B $3.5-, 2 \%$ (95\% CI: < 0.1\%-12\%) (Table 1). For those patients treated by the BP protocol, there was a significantly lower incidence of DPH than during the period prior to the $\mathrm{BP}$ protocol for SPC C patients $(\mathrm{p}=0.035)$. However, there was no significant difference in the incidence of DPH for SPC B $3.5+$ patients between these 2 periods.

\section{Comparison of Patients Who Developed DPH With Those Who Did Not Develop DPH}

A comparison of patients who experienced a DPH with those who did not (details provided in Table 2) identified the following: the mean maximum bAVM diameters were larger, the bAVMs were more likely to involve eloquent brain, the bAVMs were more often supplied by a combined source (that is, from anterior and posterior circulations), and the bAVMs were more likely to have undergone preoperative embolization. However, it must be noted that there is an obvious correlation between size and dual-circulation supply. In addition, there is a significantly higher proportion of cases embolized during the period prior to the BP protocol than for those during the BP protocol period (Table 2, Fig. 2). To see the changes that occurred to embolization and outcomes for the 4 groups of patients over the period when the cohort was surgically treated, see Fig. 2.

The outcome for patients with DPH was significantly worse than for those who did not experience DPH. Thirteen percent of patients with DPH died compared with $2.5 \%$ of patients without DPH. Presentation was typically dramatic; DPH in 4 patients was recognized after the de- velopment of reduced conscious states and a loss of pupillary response to light in patients' dilated pupils (Table 2).

\section{Complications of BP Protocol}

The main causes for permanent new neurological deficits and mortality for the 162 patients treated by the BP protocol are listed in Table 3. This list does not include reversible adverse outcomes except those who developed DPH without a subsequent permanent new neurological deficit. All cases of DPH that required surgery are reported, irrespective of eventual neurological outcome. The database would have captured mortality due to pulmonary embolus had it occurred for these cases. No patients treated by the BP protocol died, had a permanent new neurological deficit leading to an mRS score $>1$ at 12 months, or developed irreversible organ failure that could be directly attributable to the BP protocol. However, the database does not include reversible neurological complications or permanent complications that did not result in either death, neurological deficits, or permanent organ failure. Furthermore, although the permanent neurological deficit is attributed to surgery, the contribution that postoperative hypotension may have made to deficits is incalculable.

\section{Evidence of Completeness of bAVM Resection}

For the 32 patients with DPH, complete occlusion of the bAVM could only be confirmed prior to surgery for DPH in 9 cases. This was due to the DPH occurring prior to a scheduled postoperative DSA and the emergency nature of the onset of DPH. For those patients who underwent DSA prior to DPH, none were found to have residual bAVM. At time of surgery for DPH, 4 patients had further resection of bleeding, arterialized, and tense vessels. All 32 patients 
TABLE 1. Baseline characteristics, treatment, and outcomes for patients not managed by the BP protocol in the 2 time epochs

\begin{tabular}{|c|c|c|c|c|}
\hline & Variable & $\begin{array}{c}\text { Prior to BP } \\
\text { Protocol }\end{array}$ & $\begin{array}{l}\text { BP Protocol } \\
\text { Period }\end{array}$ & $\begin{array}{c}\text { Comparison } \\
\text { p Value }\end{array}$ \\
\hline \multicolumn{5}{|l|}{ Demographic data } \\
\hline \multicolumn{5}{|c|}{ Total no. of patients } \\
\hline SPC A & & 98 & 265 & \\
\hline SPC B 3.5- & & 12 & 48 & \\
\hline \multicolumn{5}{|l|}{ Female } \\
\hline SPC A & & $40(41) ; 32-51$ & 128 (48); 42-54 & 0.24 \\
\hline SPC B 3.5- & & $7(58) ; 32-81$ & $21(44) ; 31-58$ & 0.52 \\
\hline \multicolumn{5}{|c|}{ Age in yrs, mean (SD) } \\
\hline SPC A & & $40(16)$ & $39(16)$ & 0.49 \\
\hline SPC B 3.5- & & $46(17)$ & $36(16)$ & 0.06 \\
\hline \multicolumn{5}{|l|}{ bAVM } \\
\hline \multicolumn{5}{|l|}{ Infratentorial } \\
\hline SPC A & & $11(11) ; 6.2-19$ & 35 (13); 9.6-18 & 0.72 \\
\hline SPC B 3.5- & & $3(25) ; 8.3-54$ & 18 (38); 25-52 & 0.51 \\
\hline \multicolumn{5}{|c|}{ Max diameter in cm, mean (SD) } \\
\hline SPC A & & $2.7(1.2)$ & $2.4(1.2)$ & 0.07 \\
\hline SPC B 3.5- & & $2.1(0.7)$ & $2.3(0.8)$ & 0.52 \\
\hline \multicolumn{5}{|l|}{ Eloquent } \\
\hline SPC A & & $26(27) ; 19-36$ & $37(14) ; 10-19$ & $<0.01$ \\
\hline SPC B 3.5- & & $11(92) ; 62-99$ & $36(75) ; 61-85$ & 0.43 \\
\hline \multicolumn{5}{|c|}{ Deep venous drainage } \\
\hline SPC A & & $5(5.1) ; 1.9-12$ & $58(22) ; 17-27$ & $<0.01$ \\
\hline SPC B 3.5- & & $9(75) ; 46-92$ & 41 (15); 12-20 & 0.40 \\
\hline \multicolumn{5}{|c|}{ Both anterior \& posterior circulation } \\
\hline SPC A & & 15 (15); 9.4-24 & $36(14) ; 9.9-18$ & 0.73 \\
\hline SPC B 3.5- & & $2(17) ; 3.5-46$ & $11(23) ; 13-37$ & 0.99 \\
\hline \multicolumn{5}{|l|}{ Clinical } \\
\hline \multicolumn{5}{|c|}{ Preop mRS score $<2$} \\
\hline SPC A & & $57(58) ; 48-67$ & $182(69) ; 63-74$ & 0.06 \\
\hline SPC B 3.5- & & $3(25) ; 8.3-49$ & $26(54) ; 40-67$ & 0.11 \\
\hline \multicolumn{5}{|c|}{ Hemorrhage presentation } \\
\hline SPC A & & $50(51) ; 41-61$ & $130(49) ; 43-55$ & 0.81 \\
\hline SPC B 3.5- & & $10(83) ; 54-97$ & $32(67) ; 53-78$ & 0.32 \\
\hline \multicolumn{5}{|c|}{ Seizure presentation } \\
\hline SPC A & & $30(31) ; 22-40$ & 60 (23); 18-28 & 0.13 \\
\hline SPC B 3.5- & & $1(8.3) ;<0.1-38$ & $6(13) ; 5.5-25$ & $>0.99$ \\
\hline \multicolumn{5}{|l|}{ Treatment } \\
\hline \multicolumn{5}{|l|}{ Preop embol } \\
\hline SPC A & & $17(17) ; 11-26$ & $4(1.5) ; 0.5-4.0$ & $<0.01$ \\
\hline SPC B 3.5- & & $1(8.3) ;<0.1-38$ & $2(4.2) ; 0.4-15$ & 0.49 \\
\hline \multicolumn{5}{|l|}{ Outcome } \\
\hline \multicolumn{5}{|l|}{ mRS score $<2$} \\
\hline SPC A & & 88 (90); 82-95 & $232(88) ; 83-91$ & $0.16 \dagger$ \\
\hline SPC B 3.5- & & $3(25) ; 8.3-54$ & $27(56) ; 42-69$ & $<0.01 \dagger$ \\
\hline \multicolumn{5}{|l|}{ mRS score 2} \\
\hline SPC A & & 10 (10); 5.5-18 & $21(7.9) ; 5.2-12$ & \\
\hline SPC B 3.5- & & $1(8.3) ;<0.1-38$ & 14 (29); 18-43 & \\
\hline
\end{tabular}


» CONTINUED FROM PAGE 1030

TABLE 1. Baseline characteristics, treatment, and outcomes for patients not managed by the BP protocol in the 2 time epochs

\begin{tabular}{|c|c|c|c|}
\hline Variable & $\begin{array}{l}\text { Prior to BP } \\
\text { Protocol }\end{array}$ & $\begin{array}{l}\text { BP Protocol } \\
\text { Period }\end{array}$ & $\begin{array}{l}\text { Comparison } \\
\text { p Value }\end{array}$ \\
\hline \multicolumn{4}{|l|}{ Outcome (continued) } \\
\hline \multicolumn{4}{|l|}{ mRS score $3-5$} \\
\hline SPC A & $0(0) ; 0-4.5$ & (9) $3.4 ; 1.7-6.4$ & \\
\hline SPC B 3.5- & 7 (58); 32-81 & $4(8.3) ; 2.8-20$ & \\
\hline \multicolumn{4}{|l|}{ Dead } \\
\hline SPC A & $0(0) ; 0-4.5$ & $3(1.1) ; 0.2-3.4$ & \\
\hline SPC B 3.5- & $1(8.3) ;<0.1-38$ & $3(6.3) ; 1.5-17$ & \\
\hline \multicolumn{4}{|c|}{ Total complications of embol or op w/ mRS score $>1$ (including DPH) } \\
\hline SPC A & $2(2.0) ; 0.1-7.6$ & $3(1.1) ; 0.2-3.4$ & 0.61 \\
\hline SPC B 3.5- & $2(17) ; 3.5-46$ & $11(23) ; 13-37$ & $>0.99$ \\
\hline \multicolumn{4}{|c|}{$\begin{array}{l}\text { Intraop hemorrhage that led to mRS score }>1 \text { at } 12 \text { mos (excluding } \\
\text { cases of DPH) }\end{array}$} \\
\hline SPC A & $0(0) ; 0-4.5$ & $0(0) ; 0-1.7$ & \\
\hline SPC B 3.5- & $1(8.3) ;<0.1-38$ & $0(0) ; 0-8.9$ & \\
\hline \multicolumn{4}{|c|}{$\begin{array}{l}\text { Neurological deficit on emergence from anesthesia due to op that } \\
\text { led to mRS score }>1 \text { at } 12 \text { mos (excluding cases of DPH \& intraop } \\
\text { hemorrhage }\end{array}$} \\
\hline SPC A & $1(1.0) ;<0.1-6.1$ & $2(0.8) ;<0.1-2.9$ & \\
\hline SPC B 3.5- & $0(0) ; 0-28$ & $8(17) ; 8.4-30$ & \\
\hline \multicolumn{4}{|c|}{$\begin{array}{l}\text { Complication of embol that led to mRS score }>1 \text { at } 12 \text { mos (excluding } \\
\text { cases of DPH) }\end{array}$} \\
\hline SPC A & $1(1.0) ;<0.1-6.1$ & $0(0) 0-1.7$ & \\
\hline SPC B 3.5- & $0(0) ; 0-28$ & $0(0) ; 0-8.9$ & \\
\hline \multicolumn{4}{|c|}{$\begin{array}{l}\text { Vasospasm, ruptured aneurysm, or venous infarction that led to mRS } \\
\text { score }>1 \text { at } 12 \text { mos (excluding cases of } D P H)\end{array}$} \\
\hline SPC A & $0(0) ; 0-4.5$ & $0(0) ; 0-1.7$ & \\
\hline SPC B 3.5- & $1(8.3) ;<0.1-38$ & $1(2.1) ;<0.1-12$ & \\
\hline \multicolumn{4}{|c|}{ Death from acute myocardial infarction or pulmonary embolus } \\
\hline SPC A & $0(0) ; 0-4.5$ & $1(0.4) ;<0.1-2.3$ & \\
\hline SPC B 3.5- & $0(0) ; 0-28$ & $1(2.1) ;<0.1-12$ & \\
\hline \multicolumn{4}{|l|}{$\mathrm{DPH}$} \\
\hline \multicolumn{4}{|c|}{ Total DPH (including cases that did not lead to an mRS score $>1$ ) } \\
\hline SPC A & $1(1.0) ;<0.1-6.1$ & $4(1.5) ; 0.5-4.0$ & $>0.99$ \\
\hline SPC B 3.5- & $0(0) ; 0-28$ & $1(2) ;<0.1-12$ & $>0.99$ \\
\hline \multicolumn{4}{|c|}{ Complications from DPH leading to $\mathrm{mRS}$ score $>1$} \\
\hline SPC A & $0(0) ; 0-4.5$ & $1(0.4) ;<0.1-2.3$ & $>0.99$ \\
\hline SPC B 3.5- & $0(0) ; 0-28$ & $1(2.1) ;<0.1-12$ & $>0.99$ \\
\hline \multicolumn{4}{|l|}{ Follow-up in mos, median (max) } \\
\hline SPC A & $60(91)$ & $168(294)$ & $<0.01$ \\
\hline SPC B 3.5- & $75(91)$ & $157(282)$ & $<0.01$ \\
\hline
\end{tabular}

embol = embolization.

Unless otherwise noted, all values are reported as the number of patients (\%), $95 \% \mathrm{Cl}$.

* Comparisons are performed using t-, Mann-Whitney, Fisher's exact, or $\chi^{2}$ tests as appropriate. A comparison was not made for the subgroup classification of complications because there was no difference in overall complications between the 2 epochs.

$\dagger \chi^{2}$ test for outcomes.

with DPH were shown by DSA to have complete occlusion of the bAVM immediately after surgery for the DPH.

For the 609 patients who did not have DPH, 5.6\%
(95\% CI 4.0-7.7) were found to have residual bAVM, for which they either had an immediate reoperation or were discharged from the hospital without known obliteration. 
TABLE 2. Baseline characteristics, treatment, and outcomes for patients managed by the BP protocol in the 2 epochs

\begin{tabular}{|c|c|c|c|}
\hline Variable & $\begin{array}{c}\text { Prior to BP } \\
\text { Protocol }\end{array}$ & $\begin{array}{c}\text { BP Protocol } \\
\text { Period }\end{array}$ & $\begin{array}{c}\text { Comparison } \\
p \text { Value }\end{array}$ \\
\hline \multicolumn{4}{|l|}{ Demographic data } \\
\hline \multicolumn{4}{|l|}{ Total no. of patients } \\
\hline SPC B 3.5+ & 39 & 101 & \\
\hline SPC C & 17 & 61 & \\
\hline \multicolumn{4}{|l|}{ Female } \\
\hline SPC B 3.5+ & $18(46) ; 32-61$ & $56(55) ; 46-65$ & 0.35 \\
\hline SPC C & $9(53) ; 31-74$ & 31 (51); 39-63 & 0.99 \\
\hline \multicolumn{4}{|l|}{ Age in yrs, mean (SD) } \\
\hline SPC B 3.5+ & $35(13)$ & $33(14)$ & 0.56 \\
\hline SPC C & $38(16)$ & $36(16)$ & 0.73 \\
\hline \multicolumn{4}{|l|}{ bAVM } \\
\hline \multicolumn{4}{|l|}{ Infratentorial } \\
\hline SPC B 3.5+ & $2(5.1) ; 0.5-15$ & $9(8.9) ; 4.6-16$ & 0.73 \\
\hline SPC C & $2(12) ; 2.0-36$ & 4 (6.6); 2.1-16 & 0.61 \\
\hline \multicolumn{4}{|l|}{ Max diameter in $\mathrm{cm}$, mean (SD) } \\
\hline SPC B 3.5+ & $4.3(0.7)$ & $4.6(1.3)$ & 0.16 \\
\hline SPC C & $4.9(1.3)$ & $5.7(1.8)$ & 0.11 \\
\hline \multicolumn{4}{|l|}{ Eloquent } \\
\hline SPC B 3.5+ & $28(71) ; 56-84$ & $55(54) ; 45-64$ & 0.08 \\
\hline SPC C & 17 (100); 78-100 & $52(85) ; 74-92$ & 0.19 \\
\hline \multicolumn{4}{|l|}{ Deep venous drainage } \\
\hline SPC B 3.5+ & $10(26) ; 14-41$ & 41 (41); 32-50 & 0.12 \\
\hline SPC C & $14(82) ; 58-95$ & $52(85) ; 74-92$ & 0.72 \\
\hline \multicolumn{4}{|l|}{ Both anterior \& posterior circulation } \\
\hline SPC B 3.5+ & $21(54) ; 39-68$ & $41(41) ; 32-50$ & 0.19 \\
\hline SPC C & $7(41) ; 22-64$ & $38(62) ; 50-73$ & 0.17 \\
\hline \multicolumn{4}{|l|}{ Clinical } \\
\hline \multicolumn{4}{|l|}{ Preop mRS score $<2$} \\
\hline SPC B 3.5+ & $24(62) ; 46-75$ & $72(71) ; 62-79$ & 0.31 \\
\hline SPC C & 9 (53); 31-74 & $30(49) ; 37-61$ & $>0.99$ \\
\hline \multicolumn{4}{|l|}{ Hemorrhage presentation } \\
\hline SPC B 3.5+ & $15(38) ; 25-54$ & $34(34) ; 25-43$ & 0.69 \\
\hline SPC C & $7(41) ; 22-64$ & $25(41) ; 30-54$ & $>0.99$ \\
\hline \multicolumn{4}{|l|}{ Seizure presentation } \\
\hline SPC B 3.5+ & $14(36) ; 23-52$ & $42(42) ; 32-51$ & 0.57 \\
\hline SPC C & $4(24) ; 9.1-48$ & $21(34) ; 24-47$ & 0.56 \\
\hline \multicolumn{4}{|l|}{ Treatment } \\
\hline \multicolumn{4}{|l|}{ Preop embol } \\
\hline SPC B 3.5+ & $13(33) ; 21-49$ & $14(14) ; 8.3-22$ & 0.02 \\
\hline SPC C & $9(53) ; 31-74$ & $27(44) ; 33-57$ & 0.59 \\
\hline \multicolumn{4}{|l|}{ Outcome } \\
\hline \multicolumn{4}{|l|}{ mRS score <2 } \\
\hline SPC B 3.5+ & $28(72) ; 56-84$ & $68(67) ; 58-76$ & $<0.01 \dagger$ \\
\hline SPC C & $11(65) ; 41-83$ & $22(36) ; 25-49$ & $<0.01 \dagger$ \\
\hline \multicolumn{4}{|l|}{ mRS score 2} \\
\hline SPC B 3.5+ & $8(21) ; 11-36$ & $26(26) ; 18-35$ & \\
\hline SPC C & $3(18) ; 5.4-42$ & $21(34) ; 24-47$ & \\
\hline
\end{tabular}


» CONTINUED FROM PAGE 1032

TABLE 2. Baseline characteristics, treatment, and outcomes for patients managed by the BP protocol in the 2 epochs

\begin{tabular}{|c|c|c|c|}
\hline Variable & $\begin{array}{l}\text { Prior to BP } \\
\text { Protocol }\end{array}$ & $\begin{array}{l}\text { BP Protocol } \\
\text { Period }\end{array}$ & $\begin{array}{l}\text { Comparison } \\
\text { p Value }\end{array}$ \\
\hline \multicolumn{4}{|l|}{ Outcome (continued) } \\
\hline \multicolumn{4}{|l|}{ mRS score $3-5$} \\
\hline SPC B 3.5+ & $1(2.6) ;<0.1-14$ & $7(6.9) ; 3.2-14$ & \\
\hline SPC C & $1(5.9) ;<0.1-29$ & 10 (16); 9.0-28 & \\
\hline \multicolumn{4}{|l|}{ Dead } \\
\hline SPC B 3.5+ & $2(5.1) ; 0.5-18$ & $0(0) ; 0-4.4$ & \\
\hline SPC C & $2(12) ; 2.0-36$ & $8(13) ; 6.5-24$ & \\
\hline \multicolumn{4}{|c|}{$\begin{array}{l}\text { All causes of complications of embol or surgery w/ mRS score }>1 \text { at } \\
12 \text { mos (including DPH) }\end{array}$} \\
\hline SPC B 3.5+ & 7 (18); 8.7-33 & $20(20) ; 13-29$ & $>0.99$ \\
\hline SPC C & $5(29) ; 13-53$ & 27 (44); 33-57 & 0.40 \\
\hline \multicolumn{4}{|c|}{$\begin{array}{l}\text { Intraop hemorrhage that led to mRS score }>1 \text { at } 12 \text { mos (excluding } \\
\text { cases of DPH) }\end{array}$} \\
\hline SPC B 3.5+ & $1(2.6) ;<0.1-14$ & $3(3.0) ; 0.7-8.7$ & \\
\hline SPC C & $1(5.9) ;<0.1-29$ & 5 (8.2); 3.2-18 & \\
\hline \multicolumn{4}{|c|}{$\begin{array}{l}\text { Neurological deficit on emergence from anesthesia due to surgery } \\
\text { that led to mRS score }>1 \text { at } 12 \text { mos (excluding cases of DPH \& } \\
\text { intraop hemorrhage) }\end{array}$} \\
\hline SPC B 3.5+ & $1(2.6) ;<0.1-14$ & $11(11) ; 6.0-19$ & \\
\hline SPC C & $0(0) ; 0-22$ & $14(23) ; 14-35$ & \\
\hline \multicolumn{4}{|c|}{$\begin{array}{l}\text { Complication of embol that led to mRS score }>1 \text { at } 12 \text { mos (excluding } \\
\text { cases of DPH) }\end{array}$} \\
\hline SPC B 3.5+ & $0(0) ; 0-11$ & $0(0) ; 0-4.4$ & \\
\hline SPC C & $0(0) ; 0-22$ & 3 (4.9); 1.1-14 & \\
\hline \multicolumn{4}{|c|}{$\begin{array}{l}\text { Extradural or subdural hematoma that led to mRS score }>1 \text { at } 12 \text { mos } \\
\text { (excluding cases of DPH) }\end{array}$} \\
\hline SPC B 3.5+ & $0(0) ; 0-11$ & $0(0) ; 0-4.4$ & \\
\hline SPC C & $0(0) ; 0-22$ & $1(1.6) ;<0.1-9.6$ & \\
\hline \multicolumn{4}{|c|}{$\begin{array}{l}\text { Vasospasm or venous infarction that led to mRS score }>1 \text { at } 12 \text { mos } \\
\text { (excluding cases of DPH) }\end{array}$} \\
\hline SPC B 3.5+ & $1(2.6) ;<0.1-14$ & $1(1.0) ;<0.1-5.9$ & \\
\hline SPC C & $0(0) ; 0-22$ & $0(0) ; 0-7.1$ & \\
\hline \multicolumn{4}{|c|}{ Death from acute myocardial infarction or pulmonary embolus } \\
\hline SPC B 3.5+ & $0(0) ; 0-11$ & $0(0) ; 0-4.4$ & \\
\hline SPC C & $0(0) ; 0-22$ & $0(0) ; 0-7.1$ & \\
\hline \multicolumn{4}{|l|}{ DPH } \\
\hline \multicolumn{4}{|c|}{ Total DPH (including cases that did not lead to an mRS score $>1$ ) } \\
\hline SPC B 3.5+ & $5(12) ; 5.1-27$ & $11(11) ; 6.0-19$ & 0.77 \\
\hline SPC C & $5(29) ; 13-53$ & $5(8.2) ; 3.2-18$ & 0.035 \\
\hline \multicolumn{4}{|c|}{ Complications from DPH leading to mRS score $>1$} \\
\hline SPC B 3.5+ & $4(10) ; 3.5-24$ & $5(5.0) ; 1.9-11$ & 0.26 \\
\hline SPC C & $4(24) ; 9.1-48$ & $4(6.6) ; 2.1-16$ & 0.06 \\
\hline \multicolumn{4}{|l|}{ Follow-up in mos, median (max) } \\
\hline SPC B 3.5+ & $19(165)$ & $55(204)$ & 0.79 \\
\hline SPC C & $64(128)$ & $23(181)$ & 0.79 \\
\hline
\end{tabular}

Unless otherwise indicated, all values are expressed as number (\%); $95 \% \mathrm{Cl}$.

* Comparisons are performed using t-, Mann-Whitney U-, Fisher's exact, or $\chi^{2}$ tests as appropriate. A comparison was not made for the subgroup classification of complications because there was no difference in overall complications between the 2 epochs.

$\dagger \chi^{2}$ test for outcomes. 

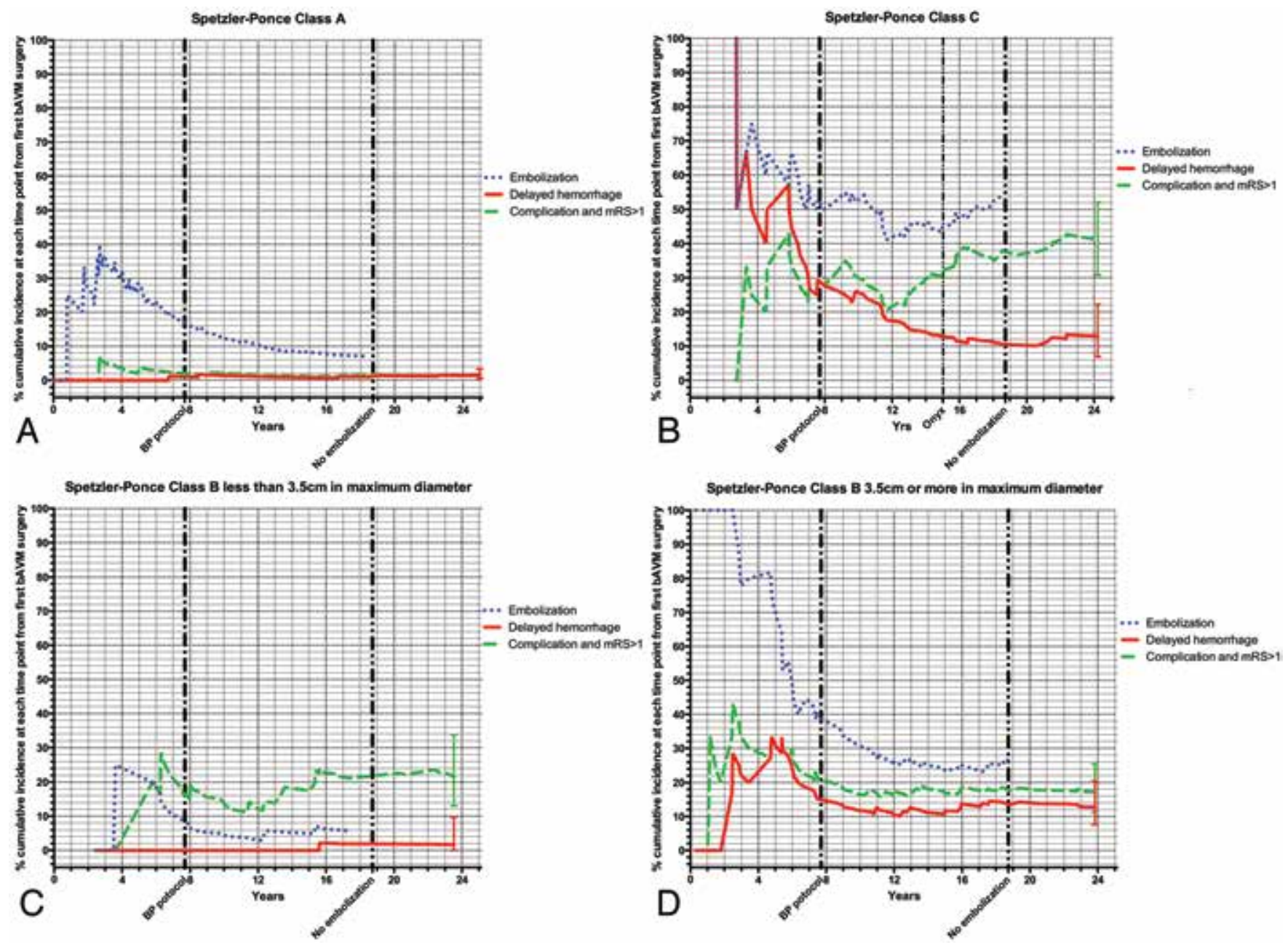

FIG. 2. The cumulative incidence of embolization (blue dots), DPH (red unbroken line), and complications leading to a permanent new neurological deficit with an mRS score $>1$ (green broken line) over time. This was calculated by dividing the total number of patients with the characteristic examined by the total number of patients to that point. The time point at which the BP protocol is indicated on each of the graphs (vertical line) is when Onyx was introduced as the embolization agent and preoperative embolization ceased.

After initial surgery or reoperation, $1.2 \%$ (95\% CI 0.5-2.4) of all patients were discharged with known residual bAVM on DSA.

\section{Regression Analysis on the Incidence of DPH for Patients With SPC B 3.5+ and SPC C bAVMs}

Details of the regression analysis are given in Table 4. Preoperative embolization was the only characteristic identified to be associated with the incidence of DPH for SPC B 3.5+ bAVMs ( $=0.013$; absence of embolization odds ratio 0.25 ; $95 \%$ CI $0.08-0.74$ ). However, for SPC C, the period prior to $\mathrm{BP}$ protocol $(\mathrm{p}=0.011$; odds ratio 7.5 ; 95\% CI 1.6-36) was significantly more likely to be associated with DPH. The absence of a combined anterior and posterior circulation contribution was weakly associated with DPH ( $\mathrm{p}=0.056$; odds ratio 0.17 ; 95\% CI $0.03-1.05$ ). Neither maximum diameter nor eloquent location was significantly associated with influencing the incidence of
DPH. Preoperative embolization was not associated with DPH for SPC C bAVMs.

\section{Change in bAVM Management Over Time}

The role of preoperative embolization significantly changed over the time of enrollment. The period during which ethylene-vinyl alcohol copolymer (Onyx) embolization was introduced is identified in Fig. 2. For those in whom embolization was used, this was performed 7 days prior to surgery. Although embolization hemorrhage occurred in 3 patients (Table 2), this preceded surgery and was not considered as DPH. In addition, there was a cumulative incidence reduction in both morbidity and DPH rates that predates introduction of the BP protocol.

It should also be noted that although the BP protocol was introduced on an identifiable date in June 1997, ${ }^{21}$ there was increasing awareness of the importance of BP management prior to this date. Therefore, during the period of 
TABLE 3. Delayed postoperative hemorrhage

\begin{tabular}{|c|c|c|c|}
\hline Variable & DPH & $\begin{array}{c}\text { No Delayed Postop } \\
\text { Hemorrhage }\end{array}$ & $\begin{array}{l}\text { Comparison } \\
\text { p Value }^{*}\end{array}$ \\
\hline Total no. of patients & 32 & 609 & \\
\hline Female & $15(47) ; 31-64$ & $295(48) ; 44-52$ & $>0.99$ \\
\hline Age in yrs, mean (SD) & $38(14)$ & $37(16)$ & 0.74 \\
\hline Max diameter in cm, mean (SD) & $4.4(1.4)$ & $3.2(1.7)$ & $<0.01$ \\
\hline Eloquent & 22 (69); 51-82 & $240(39) ; 36-43$ & $<0.01$ \\
\hline Deep venous drainage & $16(50) ; 34-67$ & $214(35) ; 31-39$ & 0.09 \\
\hline SPC A & $5(16) ; 6.4-32$ & $358(59) ; 55-63$ & $<0.01$ \\
\hline SPC B 3.5- & $1(3.1) ;<0.1-17$ & $59(9.7) ; 7.6-12$ & \\
\hline Exclusive posterior circulation & 4 (13); 4.4-29 & $168(28) ; 24-31$ & \\
\hline Both anterior \& posterior circulation & $18(56) ; 39-72$ & $153(25) ; 22-29$ & \\
\hline \multicolumn{4}{|l|}{ Clinical characteristic } \\
\hline Preop mRS score $<2$ & $21(66) ; 48-80$ & $382(63) ; 59-66$ & 0.31 \\
\hline Preop mRS score 2 & 7 (22); 11-39 & 93 (15); 13-18 & \\
\hline Preop mRS score >2 & $4(13) ; 4.4-29$ & $134(22) ; 18-27$ & \\
\hline Hemorrhage presentation & $9(28) ; 15-46$ & 294 (48); 44-52 & 0.03 \\
\hline Seizure presentation & $12(38) ; 23-55$ & $166(27) ; 24-31$ & 0.23 \\
\hline \multicolumn{4}{|l|}{ Treatment of bAVM } \\
\hline Preop embol & $12(38) ; 23-55$ & $75(12) ; 9.9-15$ & $<0.01$ \\
\hline Reoperation & $32(100)$ & NA & \\
\hline At reoperation, further resection of high pressure arterialized vessels & $2(6)$ & NA & \\
\hline \multicolumn{4}{|l|}{ DSA before \& after DPH } \\
\hline DSA before DPH & $9(28)$ & NA & \\
\hline DSA before DPH that confirmed occlusion & $9(100)$ & NA & \\
\hline DSA before discharge or death that confirmed bAVM occlusion & $32(100)$ & NA & \\
\hline \multicolumn{4}{|l|}{ Residual bAVM } \\
\hline Repeat op during hospital admission for residual identified on DSA & $0(0) ; 0-13$ & $13(2.1) ; 1.2-3.7$ & $>0.99$ \\
\hline Residual on DSA requiring repeat op or residual on discharge & $0(0) ; 0-13$ & $34(5.6) ; 4.0-7.7$ & 0.40 \\
\hline Discharge from hospital w/ known residual bAVM on DSA & $0(0) ; 0-13$ & $7(1.2) ; 0.5-2.4$ & $>0.99$ \\
\hline \multicolumn{4}{|l|}{ Outcome } \\
\hline mRS score <2 & $11(34) ; 20-52$ & $468(77) ; 73-80$ & $<0.01$ \\
\hline mRS score 2 & $14(44) ; 28-61$ & $90(15) ; 12-18$ & \\
\hline mRS score 3-5 & $3(9.4) ; 2.5-25$ & $36(5.9) ; 4.3-8.1$ & \\
\hline Dead & $4(13) ; 4.4-29$ & $15(2.5) ; 1.5-4.1$ & \\
\hline Complications of preop embol or op w/ mRS score $>1$ & 19 (59); 42-75 & $58(9.5) ; 7.4-12$ & $<0.01$ \\
\hline Days btwn op \& delayed hemorrhage, mean (SD) [median; range] & $3.3(4.1)[2 ; 0-17]$ & NA & \\
\hline
\end{tabular}

$\mathrm{NA}=$ not applicable.

Unless indicated otherwise, all values are expressed as number (\%); $95 \% \mathrm{Cl}$.

* $t-$, Mann-Whitney U-, Fisher's exact, or $\chi^{2}$ test. 
TABLE 4. Multiple logistic regressions association with DPH for SPC $C$ and SPC $b A V M \geq 3.5 \mathrm{~cm}$ maximum diameter

\begin{tabular}{|c|c|c|c|c|}
\hline \multirow[b]{2}{*}{ Characteristic } & \multicolumn{2}{|r|}{ SPC B 3.5+ } & \multicolumn{2}{|r|}{ SPC C } \\
\hline & $\mathrm{p}$ Value & Odds Ratio $(95 \% \mathrm{Cl})$ & $p$ Value & Odds Ratio $(95 \% \mathrm{Cl})$ \\
\hline No BP protocol & 0.79 & & 0.011 & $7.5(1.6-36)$ \\
\hline Excluding combined anterior \& posterior circulation supply & 0.64 & & 0.056 & $0.17(0.03-1.05)$ \\
\hline Max diameter & 0.53 & & 0.75 & \\
\hline Eloquent location & 0.78 & & 0.24 & \\
\hline No preop embol & 0.013 & $0.25(0.08-0.74)$ & 0.53 & \\
\hline
\end{tabular}

time prior to introduction of the BP protocol, there was an increasing adoption of practice that would later be defined by the BP protocol.

\section{Examination of the Cut-Point of the Association of Maximum bAVM Diameter and the Occurrence of DPH}

The ROC curve for size and the development of DPH found that the area under the curve was 0.734 (95\% CI $0.65-0.82 ; \mathrm{p}<0.01$ ) (Fig. 3). The size maximizing the addition of sensitivity and specificity was $3.2 \mathrm{~cm}$ (sensitivity $90 \%$, specificity $55 \%$ ).

\section{Discussion}

The incidence of DPH in patients with SPC A and SPC B 3.5- bAVMs is low, and patients do not seem to benefit from an aggressive BP protocol. However, these patients were in the ICU after surgery and were actively man- aged to avoid episodes of hypertension. For SPC B 3.5+ bAVMs, the incidence of DPH was significant, occurring in a little more than $10 \%$ of patients. However, for this group of bAVM, the BP protocol was not demonstrated to be associated with the prevention of DPH. For patients with SPC C, the incidence of DPH was reduced from $29 \%$ to $<10 \%$ after introduction of the BP protocol. This was a significant reduction, and regression analysis identified introduction of the BP protocol as the most significant factor associated with DPH for these SPC patients.

This study reconfirms our earlier report in 2003 of a potential benefit for aggressive BP management and further defines for whom such a benefit is likely to be realized. ${ }^{21}$ The a priori choice of a cut-point of $3.5-\mathrm{cm}$ bAVM maximum diameter for patients with SPC B bAVM seems to be reasonable from the ROC curve, which found 3.2$\mathrm{cm}$ bAVM maximum diameter to be the optimal cut-point. This result is affected by treatment that is likely to reduce

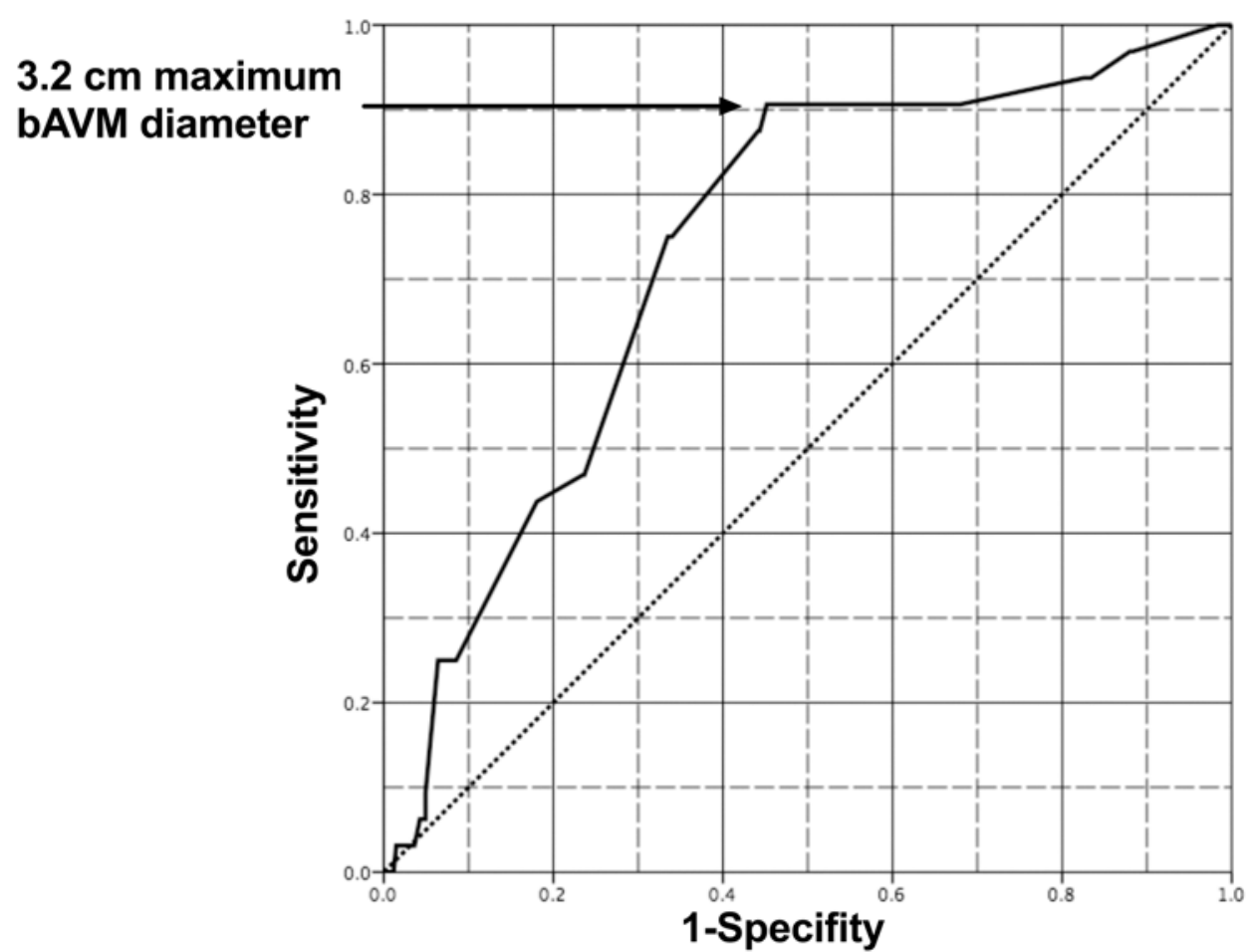

FIG. 3. An ROC curve for the association between DPH and the maximum bAVM diameter for all 641 patients undergoing microsurgery. The area under the curve was $0.734(95 \% \mathrm{Cl} 0.65-0.82 ; \mathrm{p}<0.01)$. The size maximizing the addition of sensitivity and specificity was $3.2 \mathrm{~cm}$ (sensitivity $90 \%$, specificity $55 \%$ ). 
TABLE 5. Basis for pharmacological choice incorporated in the BP protocol

\begin{tabular}{|c|c|c|c|c|}
\hline $\begin{array}{c}\text { Class of } \\
\text { Agent }\end{array}$ & BP & $\begin{array}{l}\text { Speed of Upstroke } \\
\text { in Systolic Wave }\end{array}$ & $\begin{array}{l}\text { Cardiac } \\
\text { Output }\end{array}$ & Autoregulation \\
\hline Beta-blocker & Reduction & Reduction & $\begin{array}{l}\text { Potential slight } \\
\text { reduction }\end{array}$ & No effect \\
\hline Angiotensin-converting inhibitor & Reduction & No effect & No effect & Reduces lower limit in normal brain \\
\hline Nitroprusside & Reduction w/ ability to be titrated & Increases & No effect & Slight impairment \\
\hline Calcium channel blocker & Reduction & Increases & Minimal effect & $\begin{array}{l}\text { Slight impairment but may reduce } \\
\text { impact of vasospasm }\end{array}$ \\
\hline Barbiturate & Reduction & Reduction & Reduction & $\begin{array}{l}\text { No effect but cerebral protection at } \\
\text { low BP }\end{array}$ \\
\hline Furosemide & $\begin{array}{l}\text { Reduction in event of fluid overload } \\
\text { from reduced cardiac output }\end{array}$ & No effect & $\begin{array}{l}\text { No effect or small } \\
\text { reduction }\end{array}$ & No effect \\
\hline
\end{tabular}

the occurrence of DPH for bAVM $>3.5 \mathrm{~cm}$. Therefore, the a priori choice of $3.5-\mathrm{cm}$ bAVM maximum diameter seems to be reasonable.

The BP protocol is complex and highly nuanced. An explanation of the pharmacological regimen is provided in Table 5. Treatment of normal perfusion pressure breakthrough with a reduction in BP was first proposed in $1977^{33}$ and has been demonstrated to be successful in case examples as early as $1982 . .^{10}$ Normal perfusion pressure, in the narrowest definition, is 1 of 4 potential hemodynamic mechanisms responsible for DPH. These 4 physiological mechanisms that can lead to DPH are as follows:

1. Normal perfusion pressure breakthrough caused by the inability of the microcirculation in the region of the bAVM to defend the rapid rise in pressure from low toward normal by an autoregulatory response. ${ }^{33}$ In support of this mechanism is the finding that perinidal capillaries differ significantly from those normally found in the brain, adding some evidence that they may be vulnerable to breach after removal of the arteriovenous shunt. ${ }^{27,28}$

2. Occlusive hyperemia, the consequence of propagated thrombosis in a dilated venous drainage system becoming redundant after arteriovenous fistula ablation. ${ }^{1}$

3. Rupture of small feeding arteries. The rise in pressure to levels above normal in distal feeding arteries to the former bAVM (that is, not arteries involved in supplying the microcirculation to surrounding brain) rendered thin-walled, by the long-standing low pressure within, may exceed the arterial walls' integrity.

4. Retained bAVM (see below).

A unifying mechanism for these various points of vulnerability is the arteriocapillary-venous hypertension syndrome due to the rise in pressures proximal to the point of arteriovenous fistula ablation, irrespective of where that occlusion takes place. ${ }^{19}$ That a rapid rise in BP occurs is both theoretically apparent and has also been confirmed clinically. This occurs with ${ }^{5,23}$ or without ${ }^{4,5,12,31} \mathrm{DPH}$. Although the magnitude of this rapid rise is undoubtedly important, the robustness of the wall of the artery that is subject to this rise in pressure is also of importance. This is critical when considering that the measured $\mathrm{BP}$ in these feeding arteries will compare similarly to the downstream pressures at the point of occlusion and division. This equivalence is not seen prior to vessel occlusion due to the low-resistance arteriovenous shunting that would result in downstream vessels having a considerably lower pressure than at the point of measurement. ${ }^{17}$

\section{Residual Nidus}

The evidence that residual bAVM is responsible for DPH deserves examination. Of the 32 patients with DPH, only 4 required additional resection of abnormal vessel. There was no detectable evidence of arteriovenous shunting for any of the 9 patients for whom time permitted a DSA to be performed prior to surgery for DPH. Of the patients who did not experience DPH, 5.6\% required reoperation during the hospital admission or were discharged from the hospital with residual bAVM.

Residual nidus is claimed to be a well-recognized cause of DPH. However, the meaning of nidus needs to be clearly understood. There is a natural bias among neurosurgeons to identify bAVM as a distinct, anatomically defined pathological process, akin to a tumor, due to the much larger number of tumor cases compared with bAVM cases. The word "nidus," derived from the radiological literature, reinforces this concept. However, what we are observing in the nidus (for the most part) are arteries and veins that were normal at some stage but have been altered by the physiological response to the arteriovenous fistula.

As such, the place at which the arteries are divided is not at the junction of tumor with normal artery. Rather, it is at a point along the course of an artery that is structurally altered by the hemodynamic response to the downstream arteriovenous fistula and is judged likely to be robust enough to withstand the consequent increase in pressure that results in an increase in circumferential wall tension. The transition from normal structure to increasingly abnormal structure can occur over a variable length of artery. An indirect reflection of this is that the bAVM may be compact or diffuse. For a compact bAVM, where the transition between normal arteries and significantly altered arteries occurs close to the bAVM and over a short distance, the decision on where to divide the arteries is very clear at surgery.

However, for diffuse bAVM, where the transition between normal arteries and significantly altered arteries oc- 
curs over a relatively longer length, the decision on where to divide the arteries is not as clear at surgery. Therefore, there is less certainty in such bAVMs that the division of the artery is in the correct place to ensure that the consequent increase in circumferential wall tension will be safely withstood and DPH avoided. This may account for the greater risk of surgery associated with diffuse bAVM..$^{13,15}$ Unfortunately, it was not possible to examine diffuseness in our cohort because we had not prospectively recorded this variable in our database.

The corollary of this decision making by the surgeon is that at reoperation, when residual nidus is thought to be responsible for DPH, what is actually observed are distended arterialized blood vessels that appear relatively dilated for their wall thickness, with significantly higher than expected wall tension that can easily rupture on manipulation with dissection. This is more readily explained by occluded outflow (and thus ablation of the arteriovenous shunt), but at a site too distal to a safe point at which circumferential wall tension will be safely withstood with an increase in pressure.

If arteriovenous shunting remains, it is more probable that neither the mean nor pulsatile pressure within the small dilated arteries would be sufficiently high to create this degree of circumferential wall tension that threatens the integrity of the arterial wall. Therefore, whether or not bAVM is present on postoperative DSA in cases of DPH, the region of the resection bed responsible for DPH is likely to be free of involvement of arteriovenous shunting. However, it may include small arteries that have had their downstream outflow blocked at a location too distal to prevent the arterial walls to withstand the new circumferential wall tension. At reoperation, resection of these arteries is required, reinforcing the concept of residual bAVM.

Given that the bAVM consists of the vasculature affected by the physiological response to the arteriovenous fistula, in one sense the concept that the source of hemorrhage arises from residual bAVM is correct. However, it is incorrect to assume that failure to obliterate the arteriovenous shunting is responsible for DPH. The importance of performing a DSA (if time permits) on recognizing DPH is to eliminate the future risk of rupture rather than ascertain the probable source of DPH within the resection bed.

\section{Complications of BP Protocol}

There were no deaths, permanent neurological deficits, or permanent organ failures that could be attributable to the BP protocol (Tables 1 and 2). However, reversible or nondisabling complications (e.g., infection; deep venous thrombosis; or disorders of the pulmonary, cardiovascular, renal, immune, hematological, or metabolic systems) have not been included in the database. These occurred, but it is not possible to know whether they were more common for those treated by the BP protocol than during the previous period. However, it can be safely assumed that patients who are ambulatory without intravenous and central venous access have fewer of these nondisabling complications than patients in the ICU.

Over the years since the cohort data were collected, the detection rate of deep venous thrombosis has increased with the routine adoption of postoperative venous duplex ultrasound of both upper and lower limbs on a weekly basis when in the ICU. The challenge to safely administer this complex BP protocol is extremely demanding. The pitfalls of barbiturate coma and the potential for complications of the reduction in BP require an expertise within both the nursing and medical ICU team. This expertise cannot be presumed to exist, but needs to be monitored and considered when deciding whether a BP protocol should be introduced. Therefore, the decision to manage patients in the manner described above should be based upon evidence that such a protocol achieves its intended outcome.

With respect to the lack of benefit suggested for patients with SPC B 3.5+ bAVMs, it is important to note that all patients (including those with SPC A and SPC B 3.5bAVMs) underwent postoperative management in an ICU with prevention of a systolic BP > $140 \mathrm{~mm} \mathrm{Hg}$. Therefore, those patients who are not managed by the BP protocol as defined above do have their systolic BP closely monitored and treated aggressively, although without the need for the intricacies of the BP protocol aiming to achieve a mean arterial pressure $<70 \mathrm{~mm} \mathrm{Hg}$. It would be wrong to conclude that close supervision of $\mathrm{BP}$, and appropriate action in response to its elevation, is not warranted for any postoperative bAVM patient during the first week after surgery. Furthermore, the small number of patients analyzed in this group (SPC B 3.5+) suggests that a Type II error is possible. A larger number of patients would need to be studied to confirm that there was no benefit. Therefore, for those who use an aggressive protocol in the management of SPC B 3.5+ bAVMs, we do not believe that our study supports a discontinuation of the protocol without a considerable review of outcomes in their own experience.

\section{Limitations of the Study}

The limitations of our cohort, including referral and selection bias, have been discussed previously. ${ }^{9,14}$ However, there are specific limitations that need to be discussed with regard to the current study, namely comparing 2 consecutive periods of time in cohort studies. In particular, the impact of experience of the surgeon (learning) may account for the improvement in the incidence of DPH. However, with respect to patients with an SPC A or SPC B 3.5- bAVM (the groups not treated by the BP protocol), there was no difference between the outcomes in terms of either complications or DPH when comparing the pre-BP protocol period with the BP protocol period. Therefore, the impact of learning was not evident for these groups of patients with bAVMs.

Embolization was found to be associated with an increased likelihood of DPH for patients with SPC B 3.5+ bAVMs. However, the selection of cases to be embolized was partially based on which bAVMs were thought most likely to bleed. Thus, embolization is unlikely to be the cause of DPH in patients with SPC B 3.5+ bAVMs, but rather a marker for those who are most likely to develop DPH. Examining the outcomes over time in Fig. 2 suggests that there has been a process of learning taking place. However, the small numbers of cumulative cases occurring early in the series will result in significant volatility in the incidence of adverse outcomes due to the small denominator and the wide CIs. 
From the time the BP protocol was introduced (after 166 patients and approximately 8 years after the first bAVM surgery), until the end of the cohort study, there was substantial reduction in the need for preoperative embolization, but no substantial difference in either complications of surgery leading to an mRS score $>1$ at 12 months for any of the 4 groups (SPC A, SPC B 3.5-, SPC B 3.5+, and SPC C) (Table 1). The impact of learning, from the point of view of avoiding adverse outcomes, appears to be confined to the earliest period and is not reflected in the comparison of results from the 2 epochs.

With respect to the reduction in incidence of DPH, only SPC C showed a decrease (although complications leading to an $\mathrm{mRS}$ score $>1$ did not differ between the 2 epochs for SPC C bAVM [Fig. 2]). This reduction in DPH may reflect learning. However, the importance of what might have been learned to avoid DPH does not necessarily preclude an important role for BP management. The point of division of the artery is important in the risk for the development of DPH, as discussed above. There are a number of visual clues (e.g., size, color, uniform caliber) that can assist the surgeon in judging the likely robustness of the arterial walls after ligation. This is a learned process, unique to bAVM surgery, and thus may be reflected by a reduction in incidence of DPH with time. However, there are 2 situations that may make it difficult to place a clip or ligate arteries at a safe location. These include: 1) deep feeding supply that may not be easily traced back to more proximal, upstream, thicker-walled arteries (necessitating microclips or ligation of extremely thin-walled, very small feeding arteries); and 2) feeding arteries in proximity to eloquent brain will need to be ligated and divided much closer to the bAVM than for noneloquently located bAVM (thus, always pushing the boundaries of what point might be safe). This is reflected in our results, in which eloquence was a risk factor for DPH in the univariate analysis.

Therefore, although a surgeon's learning will improve selection of the point of ligation of arteries to achieve the aim of the BP protocol (a reduction in disruptive arterial pressures), circumstance will limit the ideal choice of ligation, even when the surgeon has considerable experience.

A further limitation is the event rate of DPH (32 for the entire cohort, 16 for SPC B 3.5+, and 10 for SPC C). Although the event rate is small, it is sufficient to identify $\mathrm{BP}$ protocol as a factor of significance. However, there are too few events to exclude that other factors, in addition to the BP protocol, may have an impact on the occurrence of $\mathrm{DPH}$ within each group.

\section{Conclusions}

Although DPH is overall a rare problem, for those patients with SPC C and SPC B 3.5+ bAVMs, the incidence is $>10 \%$. The dramatic and often catastrophic presentation of DPH favors prevention (by avoiding episodes of hypertension in general and using a protocol designed to cause deliberate hypotension for selected cases), rather than attempting to reverse the often-catastrophic consequences of DPH. Our results suggest that patients with an SPC C bAVM may benefit from such a BP protocol, but this could not be shown for those with an SPC B 3.5+ bAVM. Furthermore, for patients with SPC A and SPC B 3.5-bAVMs, the decision to exclude these patients from management by the BP protocol (notwithstanding that they are managed to avoid hypertension during the postoperative period) appears to be reasonable.

\section{Acknowledgments}

We thank Dr. Elizabeth Anne Ritson, MBBS, for editorial assistance.

\section{References}

1. al-Rodhan NR, Sundt TM Jr, Piepgras DG, Nichols DA, Rüfenacht D, Stevens LN: Occlusive hyperemia: a theory for the hemodynamic complications following resection of intracerebral arteriovenous malformations. J Neurosurg 78:167-175, 1993

2. Andrews BT, Wilson CB: Staged treatment of arteriovenous malformations of the brain. Neurosurgery 21:314-323, 1987

3. Aoki N, Mizutani H: Arteriovenous malformation in the territory of the occluded middle cerebral artery with massive intraoperative brain swelling: case report. Neurosurgery 16:660-662, 1985

4. Barnett GH, Little JR, Ebrahim ZY, Jones SC, Friel HT: Cerebral circulation during arteriovenous malformation operation. Neurosurgery 20:836-842, 1987

5. Barrow DL: Unruptured cerebral arteriovenous malformations presenting with intracranial hypertension. Neurosurgery 23:484-490, 1988

6. Batjer HH, Devous MD Sr, Seibert GB, Purdy PD, Bonte FJ: Intracranial arteriovenous malformation: relationship between clinical factors and surgical complications. Neurosurgery 24:75-79, 1989

7. Bonnal J, Born JD, Hans P: One-stage excision of high-flow arteriovenous malformations. J Neurosurg 62:128-131, 1985

8. Cordato DJ, Herkes GK, Mather LE, Gross AS, Finfer S, Morgan MK: Prolonged thiopentone infusion for neurosurgical emergencies: usefulness of therapeutic drug monitoring. Anaesth Intensive Care 29:339-348, 2001

9. Davidson AS, Morgan MK: How safe is arteriovenous malformation surgery? A prospective, observational study of surgery as first-line treatment for brain arteriovenous malformations. Neurosurgery 66:498-505, 2010

10. Day AL, Friedman WA, Sypert GW, Mickle JP: Successful treatment of the normal perfusion pressure breakthrough syndrome. Neurosurgery 11:625-630, 1982

11. Drake CG: Cerebral arteriovenous malformations: considerations for and experience with surgical treatment in 166 cases. Clin Neurosurg 26:145-208, 1979

12. Hassler W, Steinmetz H: Cerebral hemodynamics in angioma patients: an intraoperative study. J Neurosurg 67:822-831, 1987

13. Kim H, Abla AA, Nelson J, McCulloch CE, Bervini D, Morgan MK, et al: Validation of the supplemented Spetzler-Martin grading system for brain arteriovenous malformations in a multicenter cohort of 1009 surgical patients. Neurosurgery 76:25-33, 2015

14. Korja M, Bervini D, Assaad N, Morgan MK: Role of surgery in the management of brain arteriovenous malformations: prospective cohort study. Stroke 45:3549-3555, 2014

15. Lawton MT, Kim H, McCulloch CE, Mikhak B, Young WL: A supplementary grading scale for selecting patients with brain arteriovenous malformations for surgery. Neurosurgery 66:702-713, 2010

16. Luessenhop AJ, Ferraz FM, Rosa L: Estimate of the incidence and importance of circulatory breakthrough in the surgery of cerebral arteriovenous malformations. Neurol Res 4:177-190, 1982 
17. Morgan M, Winder M: Haemodynamics of arteriovenous malformations of the brain and consequences of resection: a review. J Clin Neurosci 8:216-224, 2001

18. Morgan MK, Johnston IH, Hallinan JM, Weber NC: Complications of surgery for arteriovenous malformations of the brain. J Neurosurg 78:176-182, 1993

19. Morgan MK, Sekhon LHS, Finfer S, Grinnell V: Delayed neurological deterioration following resection of arteriovenous malformations of the brain. J Neurosurg 90:695-701, 1999

20. Morgan MK, Sundt TM Jr: The case against staged operative resection of cerebral arteriovenous malformations. Neurosurgery 25:429-436, 1989

21. Morgan MK, Winder M, Little NS, Finfer S, Ritson EA: Delayed hemorrhage following brain AVM resection. J Neurosurg 99:967-971, 2003

22. Mullan S, Brown FD, Patronas NJ: Hyperemic and ischemic problems of surgical treatment of arteriovenous malformations. J Neurosurg 51:757-764, 1979

23. Nornes H, Grip A: Hemodynamic aspects of cerebral arteriovenous malformations. J Neurosurg 53:456-464, 1980

24. Pertuiset B, Sichez JP, Philippon J, Fohanno D, Horn YE: [Mortality and morbidity following the surgical excision of 162 intracranial arteriovenous malformations (author's transl).] Rev Neurol 135:319-327, 1979 (Fr)

25. Rangel-Castilla L, Spetzler RF, Nakaji P: Normal perfusion pressure breakthrough theory: a reappraisal after 35 years. Neurosurg Rev 38:399-405, 2015

26. Rankin J: Cerebral vascular accidents in patients over the age of 60. II. Prognosis. Scott Med J 2:200-215, 1957

27. Sato S, Kodama N, Sasaki T, Matsumoto M, Ishikawa T: Perinidal dilated capillary networks in cerebral arteriovenous malformations. Neurosurgery 54:163-170, 2004

28. Sekhon LHS, Morgan MK, Spence I: Normal perfusion pressure breakthrough: the role of capillaries. J Neurosurg 86:519-524, 1997

29. Solomon RA, Michelsen WJ: Defective cerebrovascular autoregulation in regions proximal to arteriovenous malformations of the brain: a case report and topic review. Neurosurgery 14:78-82, 1984

30. Spetzler RF, Martin NA: A proposed grading system for arteriovenous malformations. J Neurosurg 65:476-483, 1986

31. Spetzler RF, Martin NA, Carter LP, Flom RA, Raudzens PA,
Wilkinson E: Surgical management of large AVM's by staged embolization and operative excision. J Neurosurg 67:17-28, 1987

32. Spetzler RF, Ponce FA: A 3-tier classification of cerebral arteriovenous malformations. Clinical article. J Neurosurg 114:842-849, 2011

33. Spetzler RF, Wilson CB, Weinstein P, Mehdorn M, Townsend J, Telles D: Normal perfusion pressure breakthrough theory. Clin Neurosurg 25:651-672, 1978

34. U HS: Microsurgical excision of paraventricular arteriovenous malformations. Neurosurgery 16:293-303, 1985

35. van Swieten JC, Koudstaal PJ, Visser MC, Schouten HJ, van Gijn J: Interobserver agreement for the assessment of handicap in stroke patients. Stroke 19:604-607, 1988

36. Wilson CB, Hoi Sang U, Domingue J: Microsurgical treatment of intracranial vascular malformations. J Neurosurg 51:446-454, 1979

37. Yamada K, Hayakawa T, Yoshimine T, Nakao K, Ushio Y, Mogami H: [Surgery of high-flow arteriovenous malformation: with special reference to normal perfusion pressure breakthrough phenomenon.] No Shinkei Geka 14:741-748 1986 (Jpn)

\section{Disclosures}

The authors report no conflict of interest concerning the materials or methods used in this study or the findings specified in this paper.

\section{Author Contributions}

Conception and design: Morgan. Acquisition of data: Morgan. Analysis and interpretation of data: Morgan, Wiedmann, Heller. Drafting the article: all authors. Critically revising the article: all authors. Reviewed submitted version of manuscript: all authors. Approved the final version of the manuscript on behalf of all authors: Morgan. Statistical analysis: Morgan, Heller.

\section{Correspondence}

Michael Kerin Morgan, Department of Clinical Medicine, Macquarie University, 2 Technology Pl., Macquarie University NSW 2109, Australia. email: michael.morgan@mq.edu.au. 